\title{
Efecto de la pluviosidad y el brillo solar sobre la producción y características del semen en el pez Brycon henni (Pisces: Characidae)
}

\author{
Tabares C.J. ${ }^{1}$, Montoya A.F. ${ }^{1}$, Arboleda L. ${ }^{3}$, Echeverri A. ${ }^{3}$, Restrepo L.F. ${ }^{2} \&$ Olivera-Angel M. ${ }^{1,2}$ \\ 1 Reproducción-Fisiología y Biotecnología. \\ 2 Facultad de Ciencias Agrarias, Universidad de Antioquia, Carrera 75 Nº 65-87, AA: 1226, Medellín, Colombia; Tel.: \\ +574 - 4259149. Fax:+574 - 4259149; cjbares@gmail.com; loki.asgard@gmail.com; frestrepo@agronica.udea.edu.co; \\ molivera@catios.udea.edu.co \\ 3 Escuela de Ciencias Agrarias PJIC, Carrera 48 No 7-151, AA: 4932 Medellín-Colombia; larboleda@elpoli.edu.co; \\ sabaleche@yahoo.es
}

Recibido 04-II-2005. Corregido 27-VI-2005. Aceptado 19-IX-2005.

\begin{abstract}
Effect of pluviosity and sun shine on sperm production and seminal characteristics of the fish Brycon henni (Pisces: Characidae). In Colombia the fish Brycon henni is a protected endemic species. It inhabits water bodies in coffee producing areas (700-1900 ma.s.1.; 4 $\left.355^{\prime} 56^{\prime \prime} \mathrm{N}-74^{\circ} 04^{\prime} 51^{\prime \prime} \mathrm{W} ; 18-28^{\circ} \mathrm{C}\right)$. Insufficient knowledge of its basic biology and behavior prevent the commercial culture of this promising fish. We studied the production and sperm physiology of captive males. Along a year 20 samples were taken from each of 10 males. The sample was obtained by abdominal cefalo-caudal massage and transported to the laboratory at $4{ }^{\circ} \mathrm{C}$. Except for September and October (maximum rainfall), sperm was always obtained in at least $50 \%$ of the males. Color, osmolality and $\mathrm{pH}$ were similar in all the samples. Volume, concentration, viability, motility and activation time were variable: sunshine had a positive effect on volume (Spearman $p<0.05$ ) and on sperm concentration (Spearman $\mathrm{p}<0.05$ ) while pluviosity had a negative effect on volume and viability. The proportion of ions ( $\mathrm{Na}, \mathrm{K}, \mathrm{Mg}, \mathrm{Ca}$ ) was constant along the year; $\mathrm{Na}$ being 10-fold higher than $\mathrm{K}$ and 100-fold higher than $\mathrm{Mg}$ and $\mathrm{Ca}$; however the absolute concentration of all ions was slightly higher in April and in July (with no apparent relation with the other variables analyzed). Rev. Biol. Trop. 54(1): 179-187. Epub 2006 Mar 31.
\end{abstract}

Key words: Spermiology, ion concentration, environmental conditions.

Brycon henni (Eigenmann 1913), reconocida en nombre común de Sabaleta, es una especie endémica colombiana, que habita los cuerpos de agua de zonas cafeteras comprendidos entre los 435'56" $\mathrm{N}$ y $74^{\circ} 04^{\prime} 51^{\prime}$ ' W, con temperaturas de agua que oscilan entre los 18 y los $28^{\circ} \mathrm{C}$. La pesca de esta especie está legalmente prohibida en espera de un estudio que determine la condición de posible riesgo. Debido al aprecio que las poblaciones ribereñas tienen por la Sabaleta, tanto por sus calidades gastronómicas como por su comportamiento en la pesca deportiva, se justifica iniciar estudios para su producción en cautiverio.
Strüssmann et al. (1996) y Blázquez et al. (1998), demostraron que el fotoperiodo y la pluviosidad influyen en la maduración sexual de los peces de la familia Atherinidae; y Baroiller et al. (1999) y Devlin y Nagahama (2002), demostraron que estas dos variables influyen en la diferenciación gonadal de los teleósteos en general. El brillo solar y las lluvias influyen en la función testicular, mediante la inducción de la producción de melatonina (Paniagua et al. 1990); por tanto la manipulación de estas variables ambientales podría acelerar o retrazar el funcionamiento gonadal de los peces en cautiverio para controlar así su 
espermiación (Iigo y Aida 1995, Nash 1999). El objetivo del presente trabajo fue caracterizar la producción y características del cuadro espermático de $B$. henni en cautiverio, durante un año, y asociar los resultados a las condiciones ambientales de pluviosidad y el brillo solar.

\section{MATERIALES Y MÉTODOS}

Condiciones experimentales: El estudio se realizó en la estación piscícola experimental del Politécnico Jaime Isaza Cadavid (PIJC), alimentada por la quebrada Guaracú, a $6^{\circ} 25^{\prime} \mathrm{N}$ y $75^{\circ} 39^{\prime} \mathrm{W}$ (cuenca del río Cauca). Los animales habían sido capturados del medio natural, y mantenidos en estanques de $2 \times 4 \times 0.8 \mathrm{~m}$, durante un periodo de por lo menos un año, antes de comenzar el muestreo. Se seleccionaron al azar 10 machos y se marcaron con un miccrocircuito AVID (2003) (Avid identification system). Los promedios de peso y longitud de estos ejemplares fueron, respectivamente, de $111.12 \pm 20.34$ g y $20.34 \pm 1.15 \mathrm{~cm}$. El alimento contenía un $38 \%$ de proteína según dieta diseñada para tilapia.

Toma de muestra: El proyecto se diseñó para recolectar muestras cada 15 días durante un año pero, por cuarentena de la granja, en los meses de febrero, abril, junio y noviembre sólo se pudo colectar una muestra. Previo ayuno de 24 horas, cada animal se colocó sobre una toalla húmeda, se le cubrió la cabeza con la misma toalla; se secó la zona ventral alrededor del poro genital y con masaje cráneo caudal suave se colectó el semen en un tubo de $1.5 \mathrm{ml}$. Se midió el $\mathrm{pH}$ con papel indicador (Machrey-Nagel $\mathrm{pH}$ fix 0-14) y se determinó el volumen. La muestra se transportó a $4^{\circ} \mathrm{C}$ hasta el laboratorio donde se determinó el cuadro espermático. Una vez centrifugado el semen a $2500 \mathrm{~g} \mathrm{x} 3$ minutos a $4^{\circ} \mathrm{C}$, se midió la osmolalidad del plasma seminal (Micro-osmometer type 5R Roebling) y luego, se juntaron los plasmas seminales de todos los individuos para asegurar un volumen suficiente para la medición de la composición iónica, por medio de electroforesis capilar. La concentración iónica se expresó en mM.

\section{Evaluación del cuadro espermático}

Reemplazo del plasma seminal: A partir de mediciones previas de la composición iónica del plasma seminal, se preparó una solución isosmótica de $307 \mathrm{mOsm}$ (122.8 $\mathrm{mM}$ de $\mathrm{Na} \mathrm{Cl}, 37.3 \mathrm{mM}$ de $\mathrm{KCl}, 0.87 \mathrm{mM}$ de $\mathrm{CaCl}_{2}$, $0.83 \mathrm{mM}$ de $\mathrm{MgCl}_{2}$ y $16.6 \mathrm{mM}$ de HEPES, $\mathrm{NaOH} 1 \mathrm{M}$ ), en la cual los espermatozoides permanecen inmóviles.

Movilidad y tiempo de activación: El semen diluido 1:1000 en solución isosmótica, se homogenizó con vortex por un minuto. Se depositaron $5 \mu \mathrm{l}$ de esta suspensión sobre un portaobjetos y bajo control microscópico se activó el semen con $15 \mu 1$ de solución activadora hiposmótica (agua destilada buferada con HEPES). Se determinó el porcentaje de espermatozoides que adquirieron movilidad y el tiempo de duración de la misma (tiempo en el cual el $90 \%$ de los espermatozoides activados dejó de moverse). Esta medición fue realizada siempre por el mismo observador.

Viabilidad: Cinco $\mu 1$ de Eosina-Nigrosina fueron mezclados con $1 \mu \mathrm{l}$ de semen diluido $1: 1000$, en solución isosmótica para determinar el porcentaje de viabilidad.

Morfología y concentración: Se diluyó el semen 1:500 en solución de Coffin y $1 \mu 1$ de esta dilución se fijó en metanol por 10 minutos sobre un portaobjetos, se secó al aire, se coloreó con Giemsa durante 20 minutos, se lavó con agua y se observó al microscopio a 1000X. Con la misma dilución anterior se hizo el recuento en cámara de Newbauer

Variables medioambientales: De la base de datos del IDEAM (Instituto de Hidrología, Meteorología y Estudios Ambientales) se tomó la información diaria de la precipitación $(\mathrm{mm})$ y brillo solar (horas/día), durante el periodo 
del estudio. La suma de los valores de precipitación y de horas de brillo solar de los 15 días previos a la toma de la muestra se utilizó para el análisis de su efecto sobre el cuadro espermático, bajo el supuesto de que es el efecto acumulado de estas variables es el responsable de la variación de los datos a analizar.

Análisis estadístico: Para cada variable se efectuó un análisis exploratorio descriptivo unidimensional en el tiempo. Se usó la prueba de Spearman para determinar la relación entre variables, usando el SAS System 8V.

\section{RESULTADOS}

De los 10 animales iniciales, 7 sobrevivieron hasta la finalización del ensayo. Dos machos perdieron el microcircuito transcurridos 7 meses de estudio y el otro a los 11 meses. De 200 muestras posibles se obtuvieron 80, en 96 oportunidades no hubo producción de semen y las 24 restantes correspondieron a los machos que no concluyeron el experimento. De las 80 muestras obtenidas, el $90 \%$ fue producido por 7 de los 10 machos, lo que muestra que durante 10 meses del año se encuentran machos que están produciendo semen (Fig. 1). Se observó una tendencia de todos los machos a producir semen a final y principio de año (nov-mar). El 20\% de los animales sólo produjo semen al principio del estudio, lo que correspondió con el inicio de año. Otro $20 \%$ de los machos, produjo semen sólo al principio y final del año. Un 30\% produjo semen a comienzo, mediados y finales del año y el otro $30 \%$ restante produjo semen constantemente casi todo el año en mas del $70 \%$ de las fechas donde hubo producción. Fig. 1.

Características seminales como el color (blanco cremoso), la osmolalidad del plasma $(299.9 \pm 5.2 \mathrm{mOsm} / \mathrm{kg})$ y el $\mathrm{pH}(7.5 \pm 0.5)$ son similares durante todo el año (en el cuadro 1 se presentan las características seminales a lo largo del año de estudio). Cuando los machos produjeron semen el rango osciló entre 0.8 y $5.0 \times 10^{10}$ espermatozoides $/ \mathrm{ml}$ y el volumen entre 3 y $2200 \mu$, siendo los meses en que produjeron mayor volumen: noviembre, enero, marzo, mayo y diciembre. El porcentaje de espermatozoides móviles estuvo entre 5 y $90 \%$, con un tiempo de activación de $62 \pm 15.8 \mathrm{~s}$. Se encontró una relación directa entre el volumen y el porcentaje de movilidad (Spearman $\mathrm{p}<0.05$ ) y el volumen con el tiempo de activación (Spearman $\mathrm{p}<0.05)$. La viabilidad osciló entre el 12 y el $100 \%$. La morfología de la cabeza es rabdiformes en un 60 a $100 \%$ de los espermatozoides.

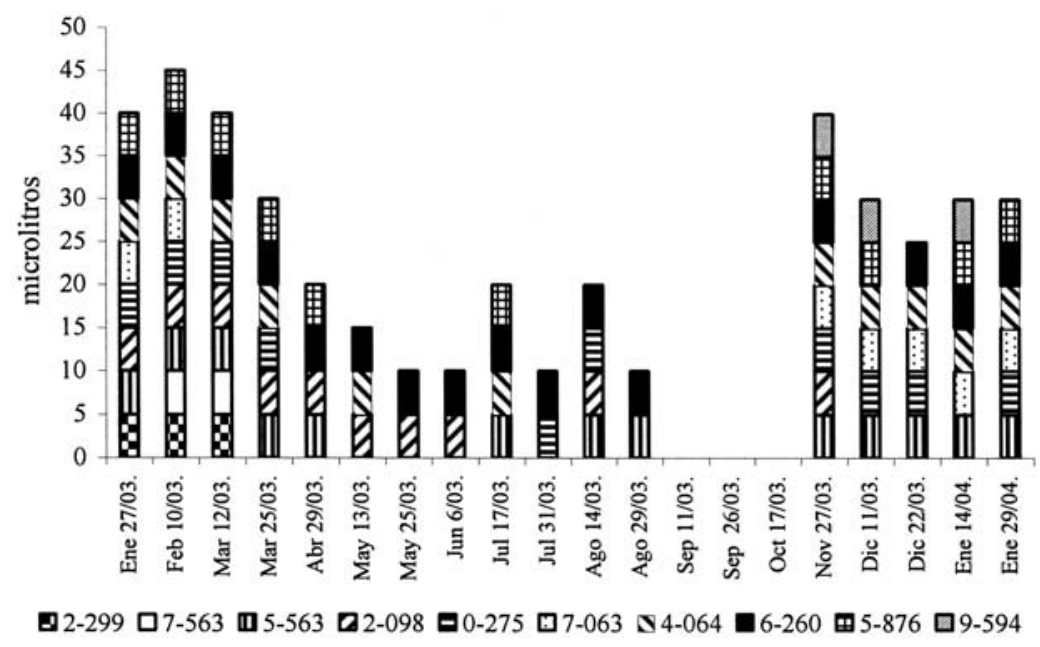

Fig. 1. Producción seminal individual en machos de B.henni.

Fig. 1. Individual seminal production frorn $B$. henni individuals. 
CUADRO 1

Características seminales de B. henni a lo largo de un año de estudio

TABLE 1

Seminal characteristics of $\mathrm{B}$. henni, along the year of study

\begin{tabular}{|c|c|c|c|c|c|c|c|c|}
\hline \multirow[b]{2}{*}{ Fecha } & \multicolumn{8}{|c|}{ Primer semestre } \\
\hline & Ene $27 / 03$ & Feb 10/03 & Mar 12/03 & Mar 25/03 & Abr $29 / 03$ & May 13/03 & May $25 / 03$ & Jun $6 / 03$ \\
\hline $\mathrm{N}$ & $8 / 10$ & $9 / 10$ & $5 / 10$ & $6 / 10$ & $4 / 10$ & $3 / 10$ & $2 / 10$ & $2 / 10$ \\
\hline T Ac. (seg.) & $40-114$ & $38-78$ & $38-66$ & $53-66$ & $43-69$ & $46-62$ & $40-53$ & $64-79$ \\
\hline Movilidad (\%) & $30-85$ & $10-80$ & $30-50$ & $10-60$ & $10-40$ & $50-90$ & $60-60$ & $5-30$ \\
\hline Volumen $(\mu \mathrm{l})$ & $3-200$ & $20-150$ & $50-500$ & $100-550$ & $10-90$ & $50-900$ & $100-480$ & $50-250$ \\
\hline $\begin{array}{l}\text { Concentración } \\
\text { (esp X } 10^{10 / m l)}\end{array}$ & $1.7-2.6$ & $1.9-3.3$ & $1.9-35$ & $1.8-3$ & $1.6-2.6$ & $1.5-2.9$ & $2.3-3.3$ & $1.6-2.9$ \\
\hline Viabilidad (\%) & $35-95$ & $32-85$ & $23-98$ & $15-98$ & $27-84$ & $47-58$ & $25-62$ & $33-78$ \\
\hline \multirow[t]{2}{*}{ Rabhdiform (\%) } & $58-96$ & 0 & $84-96$ & $96-100$ & $69-95$ & $62-87$ & $98-99$ & $80-81$ \\
\hline & \multicolumn{8}{|c|}{ Segundo semestre } \\
\hline Fecha & Jul 17/03 & Jul 31/03 & Ago 14/03 & Nov $27 / 03$ & Dic 11/03 & Dic $22 / 03$ & Enero14/04 & En $29 / 04$ \\
\hline $\mathrm{N}$ & $4 / 10$ & $2 / 10$ & $4 / 8$ & $8 / 8$ & $6 / 7$ & $5 / 7$ & $6 / 7$ & $6 / 7$ \\
\hline T.Ac. (seg) & $52-60$ & $60-70$ & $45-55$ & $55-77$ & $56-89$ & $50-73$ & $48-120$ & $58-91$ \\
\hline Movilidad (\%) & $10-50$ & $46-65$ & $50-80$ & $40-90$ & $10-40$ & $5-50$ & $5-50$ & $20-60$ \\
\hline Volumen $(\mu \mathrm{l})$ & $20-50$ & $30-110$ & $40-250$ & $25-2200$ & $50-450$ & $10-350$ & $50-600$ & $200-800$ \\
\hline $\begin{array}{l}\text { Concentración } \\
\text { (esp X } 10^{10 / m l)}\end{array}$ & $1.8-3$ & $2.5-2.6$ & $0.8-2.5$ & $1.8-4.2$ & $1.2-1.9$ & $0.8-2.6$ & $0.9-2.7$ & $2.7-5$ \\
\hline Viabilidad (\%) & $26-47$ & $58-69$ & $38-59$ & $78-95$ & $80-95$ & $77-96$ & $12-100$ & $65-100$ \\
\hline Rabhdiform (\%) & $79-98$ & $72-79$ & 89-99 & $93-100$ & $97-99$ & $94-100$ & $96-100$ & $84-99$ \\
\hline
\end{tabular}

El balance iónico entre los cuatro iones estudiados mostró la misma proporcionalidad a lo largo del año. El sodio es el mayor componente, seguido por el potasio 10 veces menor y magnesio y calcio 100 veces menores; con promedios de $\mathrm{Na}^{+}$fue de $255 \pm$ $101 \mathrm{mM}, \mathrm{K}^{+} 27.8 \pm 18, \mathrm{Ca}^{++} 2.3 \pm 0.9$, $\mathrm{Mg}^{++} 5.2 \pm 2.1$. La concentración iónica del $\mathrm{Na}^{+}$presentó diferencias significativas con el $\mathrm{K}^{+}$(Spearman $\left.\mathrm{p}<0.05\right)$, el $\mathrm{Ca}^{++}$y el $\mathrm{Mg}^{++}$ (Spearman $\mathrm{p}<0.0001)$. La concetración iónica del $\mathrm{K}$ también presentó diferencias con el el $\mathrm{Ca}^{++}$y el $\mathrm{Mg}^{++}$(Spearman $\left.\mathrm{p}<0.0001\right)$, finalmente el $\mathrm{Ca}^{++}$con el $\mathrm{Mg}^{++}$(Spearman $\mathrm{p}<0.000$ ), las concentraciones mensuales de los diferentes iones se muestran en la figura
2). No se encontró relación entre el balance iónico y los parámetros seminales.

El brillo solar afectó positivamente el volumen (Spearman $\mathrm{p}<0.05$ ) (Fig. 3) y la concentración espermática (Spearman $\mathrm{p}<0.05) \mathrm{La}$ precipitación mostró una relación inversa con el volumen (Spearman $\mathrm{p}<0.0001$ ) (Fig. 4), y la viabilidad (Spearman $\mathrm{p}<0.005$ ).

\section{DISCUSIÓN}

Los resultados del presente estudio demuestran que en cautiverio se pueden encontrar machos maduros 10 meses del año, al contrario de la $B$. siebenthalae que es estacional, 


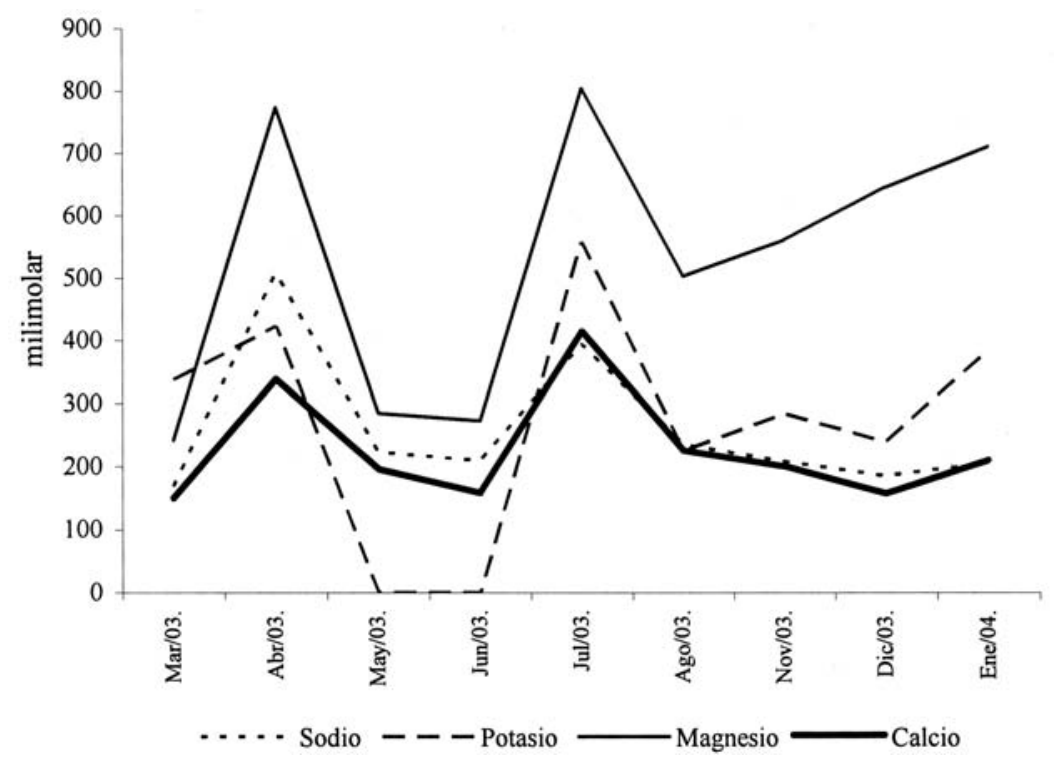

Fig. 2. Comportamiento del balance iónico a lo largo de un año. Se trasformaron los datos usando factores para ajustarlos a una misma escala $\left(\mathrm{K}^{+} \mathrm{x} 10, \mathrm{Ca}^{++} \mathrm{y} \mathrm{Mg}^{++} \mathrm{x} 100\right)$.

Fig. 2. Pattern of the ionic balance in seminal plasma of $B$. henni along the year under study. Data were transformed using factors to keep the same scale: $\left(\mathrm{K}^{+} \times 10, \mathrm{Ca}^{++} \mathrm{y} \mathrm{Mg}^{++} \times 100\right)$.

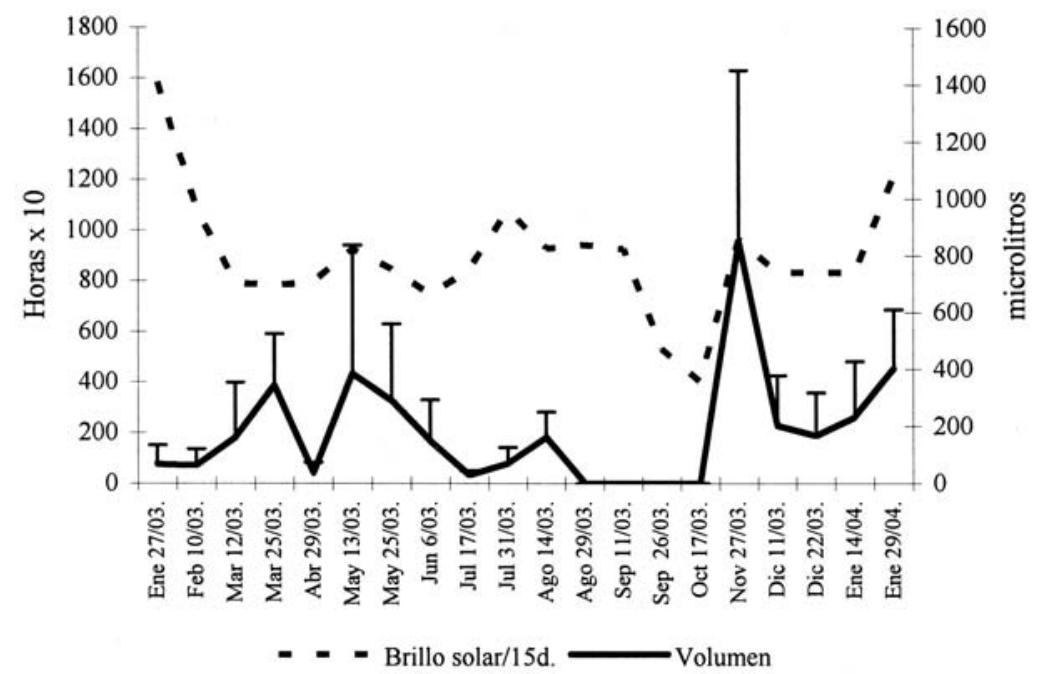

Fig. 3. Producción seminal de B. henni durante un periodo de un año vs brillo solar. El brillo solar se ajusto a la escala del volumen multiplicando por un factor de 10 .

Fig. 3. Semen production in $B$. henni along one year and association with sun shine. This last datum was adjusted to the volume scale by multiplying by 10 . 


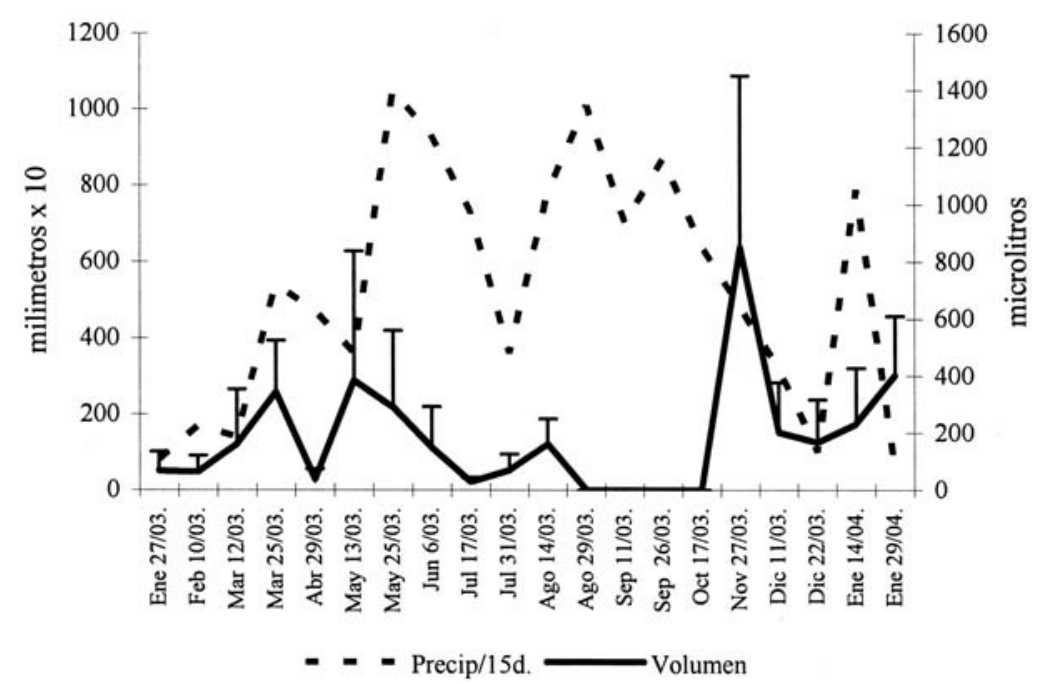

Fig. 4. Producción seminal de B.henni durante un periodo de un año vs precipitación. La precipitación se ajusto a la escala del volumen multiplicando por un factor de 10.

Fig. 4. Semen production in $B$. henni along one year and association with pluviosity. This last datum was adjusted to the volume scale by multiplying by 10 .

limitada a 4 meses (febrero-mayo). El volumen de semen de $B$. henni es similar al de $B$. siebenthalae $(1.82 \pm 0.25 \mathrm{ml})$ reportado por Cruz 2001. La concentración espermática de $B$. henni dobla la concentración de $B$. siebenthalae $\left(13.9 \pm 4 \times 10^{9} \mathrm{esp} . / \mathrm{ml}\right)$ reportada por Cruz 2001 y es inferior a la reportada por Fresneda et al. 2004 en la nativa Piaractus brachypomus $\left(3.0 \pm 1.1 \times 10^{10} \mathrm{esp} . / \mathrm{ml}\right)$.

El número de espermas móviles en $B$. siebenthalae es mayor y con menor desviación $(82 \pm 9 \%)$ (Cruz 2001) que en B. henni, (40 \pm $23 \%$ ); otros characidos presentan mayor movilidad: Brycon insignis, 90.1 a 91.7\% (AndradeTalmelli et al. 2001), Oncorhynchus mykiss, 100\% (Geffen y Evans 2000) y Plecoglossus altivelis, $89.4+/-2.1 \%$ (Ohta et al. 2001). Es posible que la variación en los porcentajes de movilidad encontrados en los machos de $B$. henni en cautiverio, se deba a factores que han reportado autores como Rurangwa et al. (2000), como son el estado nutricional, la calidad del agua y el estrés. El tiempo de activación para $B$. henni (35-62 s) fue similar al de B. siebenthalae (41.4 $\pm 7 \mathrm{~s})$ (Cruz 2001) y con un rango mas estrecho del reportado para Piaractus brachypomus (50 - 257 s) (Caleño 1995).
En el Cuadro 2 se comparan los hallazgos del presente trabajo con la concentración y proporción iónica de otros teleósteos, demostrando la diferencia en concentraciones y proporciones. Según Kraznai et al. 2000 los iones juegan un papel importante en el porcentaje de espermatozoides que inician la movilidad, sin embargo no se pudo demostrar estadísticamente esta asociación, ya que no hubo efecto de ninguno de los iones, ni de su relación con el porcentaje de movilidad, ni con el tiempo que dura ésta. El cambio en la concentración iónica que se da en los meses de abril y julio no corresponde ni con cambios climáticos, ni con cambios en las características espermáticas. Se propone estudiar las características fisicoquímicas del agua durante un periodo largo de tiempo.

La osmolalidad permanece constante durante todo el tiempo en que hubo muestra, esto quiere decir que el epitelio del conducto espermático principal es capaz de regularla bajo cualquier condición ambiental. Al igual que Morisawa (1985) reportó para otros teleosteos, podemos decir que en esta especie la isosmolalidad es la responsable de que los espermas en el plasma seminal estén quiescentes, asegurando 
CUADRO 2

Comparación de la composición iónica del plasma seminal entre diferentes especies

TABLE 2

Comparison of ionic composition of seminal plasma among different species

Composición iónica del plasma seminal en mM

\begin{tabular}{lccccl}
\multicolumn{1}{c}{ Especie } & $\mathrm{Na}^{+}$ & $\mathrm{K}^{+}$ & $\mathrm{Ca}^{++}$ & $\mathrm{Mg}^{++}$ & \multicolumn{1}{c}{ Referencia } \\
Plecoglossus altivelis & 130 & 11.4 & 0.6 & 2.3 & Ohta et al. 2001 \\
Oncorhynchus mykiss & 122.2 & 30,4 & 1.10 & 0.85 & Glogowski et al. 2000 \\
Cyprinus carpio & 55 & 82.4 & 2 & 0.8 & Ohta y Tsuji 1998 \\
Brycon henni & 255 & 27.8 & 2.03 & 5.1 &
\end{tabular}

el potencial de movilidad para la fertilización del oocito.

En el trópico se han reportado especies que desovan en épocas de lluvias ( $P$. brachypomus, B siebenthalae, Brycon bicolor, Brycon whitei y Brycon moorei sinuensis (Taphorn 1992, Otero et al. 1986) y especies que se reproducen en épocas secas (Carrillo y Rodriquez 2001). En carácidos que se reproducen en épocas de lluvias, la producción de melatonina está inhibida por que no hay estímulos lumínicos que induzcan su síntesis, por lo tanto, no hay inhibición del funcionamiento del eje hipotálamo hipófisis (Zanuy y Carrillo 1997). En el caso de B. henni (Perdomo 1978) y de B. insigni (Andrade et al. 2001), especies desovadoras durante la estación seca (diciembre y febrero) se podría pensar en dos hipótesis: la primera es que haya un efecto inverso de la melatonina sobre el eje hipotálamo hipófisis, favoreciendo la reproducción en vez de inhibirla y la segunda estaría asociada a las características físico-químicas del agua dada por la estación seca. Sin embargo en los machos de $B$. henni la luminosidad (Fig. 3) no es definitiva para la producción de semen, ya que aunque es marcado el aumento en el volumen en los meses de mayor brillo solar, se encuentra semen durante 10 meses del año. Al contrario de los reportado por Builes y Uran 1974 y Florez 1999, las lluvias afectaron negativamente el volumen del esperma, tanto que ningún macho produjo semen en los meses de septiembre y octubre cuando la precipitación fue muy alta (Fig. 4). Es posible que la calidad del agua con un aumento de la turbidez y disminución del oxígeno sea la causa de la aspermia en dos meses y la baja viabilidad asociada con la pluviosidad.

Proponemos que en $B$. henni, el comportamiento reproductivo de los machos en cautiverio, podría obedecer mas a cambios en las características fisico-químicas del agua asociadas a la estación climática que, a efectos en la producción de melatonina, apoyados en que para la reproducción en el medio silvestre, esta especie requiere aguas tranquilas, claras y poco profundas (Comunicación personal, pescadores San Jerónimo).

\section{AGRADECIMIENTOS}

A Colciencias proyecto: 1115-09-12308, Universidad de Antioquia y P C J I por la cofinanciación del proyecto.

\section{RESUMEN}

El pez Brycon henni es una especie endémica protegida por la legislación colombiana, que habita cuerpos de agua de zonas cafeteras (700-1900 m.s.n.m), comprendidas entre $\operatorname{los} 4^{\circ} 35^{\prime} 56^{\prime \prime} \mathrm{N}$ y $74^{\circ} 04^{\prime} 51^{\prime}$ " W, con temperaturas que oscilan entre los 18 y los $28^{\circ} \mathrm{C}$. A pesar de las características promisorias de esta especie, su reproducción en 
cautiverio a nivel comercial no ha sido posible por falta de conocimientos básicos de su biología y comportamiento. El objetivo del presente trabajo fue caracterizar la producción y la fisiología espermática de machos en cautiverio. A lo largo de un año se tomaron 20 muestras de cada uno de 10 ejemplares. El semen se obtuvo mediante masaje abdominal cráneo-caudal y se transportó a $4^{\circ} \mathrm{C}$ para su análisis en el laboratorio. Con excepción de septiembre y octubre que fueron los meses más lluviosos, siempre se obtuvo semen de al menos el $50 \%$ de los animales. El color, la osmolalidad y el $\mathrm{pH}$ fueron similares en todas las muestras a lo largo del año. El volumen, la concentración, la viabilidad, la movilidad y el tiempo de activación fueron variables: El efecto del brillo solar fue positivo sobre el volumen (Spearman $\mathrm{p}<0.05)$ y sobre la concentración espermática (Spearman $\mathrm{p}<0.05)$, mientras que la pluviosidad tuvo un efecto negativo sobre el volumen y la viabilidad. La proporción entre los iones $(\mathrm{Na}, \mathrm{K}, \mathrm{Mg}, \mathrm{Ca}$ ) se mantuvo constante durante todo el año: el sodio fue el mayor, seguido por el potasio, y por el magnesio y el calcio, 10 y 100 veces menos, respectivamente; sin embargo la concentración absoluta de estos iones aumentó durante los meses de abril y julio sin una relación aparente con las otras variables analizadas.

Palabras clave: Espermiología, concentración iónica, condiciones ambientales.

\section{REFERENCIAS}

Andrade-Talmelli, E.F., E.T. Kavamoto \& N. FenerichVerani. 20001. Características seminais da piabanha, Brycon insignis (Steindachner, 1876), após estimulação hormonal. Bol. Inst. Pesca, São Paulo 27(2): 149-154.

Blázquez, M., S. Zanuy, M. Carrillo \& F. Piferrer. 1998. Effects of rearing temperatures on sex differentiation in the European sea bass (Dicentrarchus labrax L.). J. Exp. Zool. 281: 207-216.

Baroiller, J.F., Y. Guiguen \& A. Fostier. 1999. Endocrine and environmental aspects of sex differentiation in fish. Cell. Mol. Life Sci. 55: 910-931.

Builes, J \& A. Uran. 1974 Estudio del ciclo sexual de la Sabaleta (B. henni) su comportamiento y fecundacion artificial. Rev. Act. Biol. 3: 7-7.

Caleño, O. 1995. Pruebas de fertilidad con semen de Cachama Blanca (Piaractus brachypomus, Cuvier, 1818) criopreservado mediante dos extendidos diferentes. Tesis de Biólogo Marino, Universidad Jorge Tadeo Lozano, Bogotá, Colombia. 131 p.

Carrillo, M. \& J. Rodríguez. 2001. Bases fisiológicas de la reproducción de peces tropicales p. 189216. In H. Rodriquez, P.V. Daza \& M. Carrillo (eds.). Fundamentos de Acuicultura Continental. Grafimpresos Quintero, Bogotá, Colombia.

Cruz, P. 2001. VIII Jornada de Acuicultura. Universidad de los Llanos, Villavicencio, Colombia. 74 p.

Devlin, R.H. \& Y. Nagahama. 2002. Sex determination and sex differentiation in fish: an overview of genetic, physiological, and environmental influences. Aquaculture 208: 191-364.

Florez, P. 1999. Estudio biológico-pesquero preliminar de tres especies ícticas del Alto Río Cauca Embalse de Salvajina. Cespedecia 23: 47-60.

Fresneda, A., G. Lenis, E. Agudelo \& M. Olivera-Angel. 2004. Espermiación inducida y crioconservación de semen de Cachama Blanca (Piaractus brachypomus). Rev. Col. Cienc. Pec. Vol. 17 (s): 46-52.

Geffen, A.J. \& J.P. Evans. 2000. Sperm traits and fertilization success of male and sex-reversed female rainbow trout Oncorhynchus mykiss. Aquaculture 182: 61-72.

Glogowski J., M. Kwasnik, K. Dabrowski, K. Goryczjo, S. Dobosz, H. Kuzmininski \& A. Ciereszko. 2000. Characterization of Rainbow trout milt collected with a catheter: semen parameters and cryopreservation success. Aquac. Res. 31: 289-296.

Iigo, M. \& K. Aida. 1995. Effects of season, temperature and photoperiod on plasma melatonin rhythms in the goldfish (Carassius auratus). J. Pineal Res. 18: 62-68.

Krasznai, Z., T. Marian, H. Izumi, S. Damjanovich, L. Balkay, L. Tron \& M. Morisawa. 2000. Membrane hyperpolarization removes inactivation of $\mathrm{Ca} 2+$ channels, leading to $\mathrm{Ca} 2+$ influx and subsequent initiation of sperm motility in the common carp. PNAS. 97: 2052-2057.

Morisawa, M. 1985. Initiation mechanism of sperm motility at spawning in teleosts. Zool. Sci. 2: 605-615.

Nash, J.P. 1999. Seasonal reproduction in fish, Vol. 4, p 329340. In Knobil, E., Neíl , J.D. (eds.). Encyclopaedia of Reproduction. Academic, Nueva York.

Ohta, H., T. Unuma, M. Tsuji, M. Yoshioka \& M. Kashiwagi. 2001 Effects of bicarbonate ions and $\mathrm{pH}$ on acquisition and maintenance of potential for motility in ayu, Plecoglossus altivelis Temminck et Schlegel (Osmeridae), spermatozoa. Aquac. Res. 32: 385-392.

Ohta, H. \& M. Tsuji. 1998. Ionic environment necessary for maintenance of potential motility in the common carp spermatozoa during in vitro storage. Fish Sci. 64: $547-552$.

Otero, F., J. Solano, A. Gonzalez \& F. Zapata. 1986. Migraciones de peces del río Sinú, Proyecto 
hidroelectrico alto Sinú. Universidad de Cordoba, Monteria, Colombia. 126 p.

Paniagua, R., B. Fraiie \& J. Sáez. 1990. Effects of photoperiod and temperature on testicular function in amphibians. Hist. Histopat. 5: 365-378.

Perdomo, J. 1978. La sabaleta: observaciones biológicas y su importancia como especie de cultivo. Rev. Div. Pesq. 11(1): 89-97.

Rurangwa, E., D. Kime, F. Ollevier \& J. Nash. 2004. The measurement of sperm motility and factors affecting sperm quality in cultured fish. Aquaculture 234: 1-28.
Strüssmann, C.A., S. Moriyama, E.F. Hanke, J.C.C. Cota \& F. Takashima. 1996. Evidence of thermo labile sex differentiation in pejerrey. J. Fish Biol. 48: 643-651.

Taphorn, D. 1992. The Characiforms fishes of the Apure river drainage, Venezuela. Biollania, Venezuela. $537 \mathrm{p}$.

Zanuy, S. \& M. Carrillo. 1997. VI Congreso Nacional de Acuicultura. Universidad de Murcia (U.M), Consejería de Medio Ambiente, Agricultura y Agua de la Comunidad Autónoma de la Región de Murcia (C.M.A.A.A.), Centro Oceanográfico de Murcia del Instituto Español de Oceanografía (I.E.O.), Ayuntamiento de Cartagena. Cartagena, Murcia. 1-7 p. 
